\title{
Secondary mechanisms by which SARS-CoV-2 affects the brain
}

\author{
Josef Finsterer, ${ }^{1}$ (iD Fulvio Alexandre Scorza, ${ }^{2}$ (iD Carla Alessandra Scorza, ${ }^{2}$ (iD \\ Ana Claudia Fiorini ${ }^{3,4}$ iD \\ ${ }^{1}$ Klinik Landstrasse, Messerli Institute, Vienna, Austria. ${ }^{2}$ Disciplina de Neurociência, Escola Paulista de Medicina/Universidade Federal de São \\ Paulo (EPM/UNIFESP), São Paulo, SP, Brazil. ${ }^{3}$ Programa de Estudos Pós-Graduados em Fonoaudiologia, Pontifícia Universidade Católica de \\ São Paulo, São Paulo, SP, Brazil. ${ }^{4}$ Departamento de Fonoaudiologia, EPM/UNIFESP, São Paulo, SP, Brazil.
}

For almost 2 years, the world has been obsessed with the pandemic triggered by the severe acute respiratory syndrome coronavirus 2 (SARS-CoV-2). Though the infection predominantly affects the lungs (coronavirus disease 2019, COVID-19), it is actually a multisystem disease, capable of affecting all organs, systems, or tissues, although some are preferentially involved. The second most frequently affected system, after the lungs, is the nervous system. The central nervous system (CNS) and peripheral nervous system (PNS), including the autonomic nervous system, can be involved. CNS manifestations of SARS-CoV-2 infections include encephalitis, meningitis, cerebellitis, acute hemorrhagic necrotizing encephalitis (AHNE), Bickerstaff brainstem encephalitis (BBE), optic neuritis, immune encephalitis, limbic encephalitis, hypophysitis, ventriculitis, ischemic stroke, intraparenchymal bleeding, subarachnoid hemorrhage, venous sinus thrombosis (VST), epilepsy, myoclonus syndrome, tremor, serotonin-like syndrome, sleep disorder, posterior reversible encephalopathy syndrome, cerebral vasculitis, giant cell arteritis, headache, migraine, insomnia, vasoconstriction syndrome, multiple sclerosis, neuromyelitis optica (NMO)-spectrum disorders, and transverse myelitis. ${ }^{1}$ PNS manifestations of SARS-CoV2 infections include the polyradiculitis Guillain-Barré syndrome (GBS), with all its subtypes (acute inflammatory demyelinating polyneuropathy, acute motor axonal neuropathy, acute motor and sensory axonal neuropathy, Miller-Fisher syndrome, pharyngo-cervico-brachial variant, polyneuritis cranialis, mononeuritis cranialis, optic neuritis, BBE), Parsonage-Turner syndrome, small fiber neuropathy, myasthenia, myasthenic syndrome, myositis, and dermatomyositis. ${ }^{1}$ Most of these conditions have been only rarely reported, but they are nonetheless interesting as models for studying the pathophysiological background of neuro-COVID.

Psychiatric disorders associated with SARS-CoV-2 include psychosis, ${ }^{2}$ hyper- or hypokinetic delirium, ${ }^{3}$ akinetic mutism, ${ }^{4}$ catatonia, ${ }^{5}$ depression, and acute confusion. The latter can be even the presenting manifestation of a SARS-CoV-2 infection. Psychiatric disease in

Correspondence: Josef Finsterer J, Postfach 20, 1180, Vienna, Austria.

E-mail: fifigs1@yahoo.de

Submitted Aug 27 2021, accepted Oct 18 2021, Epub Jan 192022.
COVID-19 patients with cerebral involvement is not uncommon. In a cross-sectional surveillance study of 267 adult hospitalized patients with COVID-19 from the United Kingdom during the first wave of the pandemic, $25 \%$ of the included patients had experienced psychiatric disease. The frequency of delirium in this cohort was $28 \%$.

Various pathophysiological concepts have been proposed to explain how the virus affects the CNS or PNS. Direct or indirect pathophysiological mechanisms can be delineated respectively. Direct mechanisms rely on the confirmed presence of the virus in the CNS or PNS and the invasiveness and toxicity of the virus. Secondary pathophysiological mechanisms explaining the development of CNS or PNS disease are by far more frequent than primary pathophysiological mechanisms, and include the host immune response to viremia, hypercoagulability, and the infection of tissues, such as endothelial cells or cardiomyocytes, by the virus, leading to secondary disease (Figure 1).

The immune response to the virus involves both the humoral system (cytokine storm, cytokine release syndrome, hemophagocytic syndrome) and the cellular system. Diseases attributable to the immune response against SARS-CoV-2 include immune encephalitis, BBE, acute disseminated encephalomyelitis, multiple sclerosis, NMO-spectrum disorder, cerebral vasculitis, transverse myelitis, polyneuritis cranialis, mononeuritis cranialis, GBS, myositis, myasthenia, myasthenic syndrome, immune thrombocytopenia, autoimmune hepatitis, immune-mediated keratolysis, autoimmune myocarditis, autoimmune hemolytic anemia, autoimmune thyroid disease, and Kawasaki syndrome.

The second pathophysiological mechanism explaining CNS/PNS involvement is hypercoagulability. Several mechanisms have been proposed to explain this hypercoagulable state. First, involvement of the endothelial cells - there is evidence of endotheliitis in COVID-19 resulting in upregulation of van Willebrand factor and factor VIII. Second, if involvement of the lungs is complicated by hypoxemia, vasoconstriction may ensue,

How to cite this article: Finsterer J, Scorza FA, Scorza CA, Fiorini AC. Secondary mechanisms by which SARS-CoV-2 affects the brain. Braz J Psychiatry. 2022;44:242-244. http://dx.doi.org/10.1590/ 1516-4446-2021-2268 


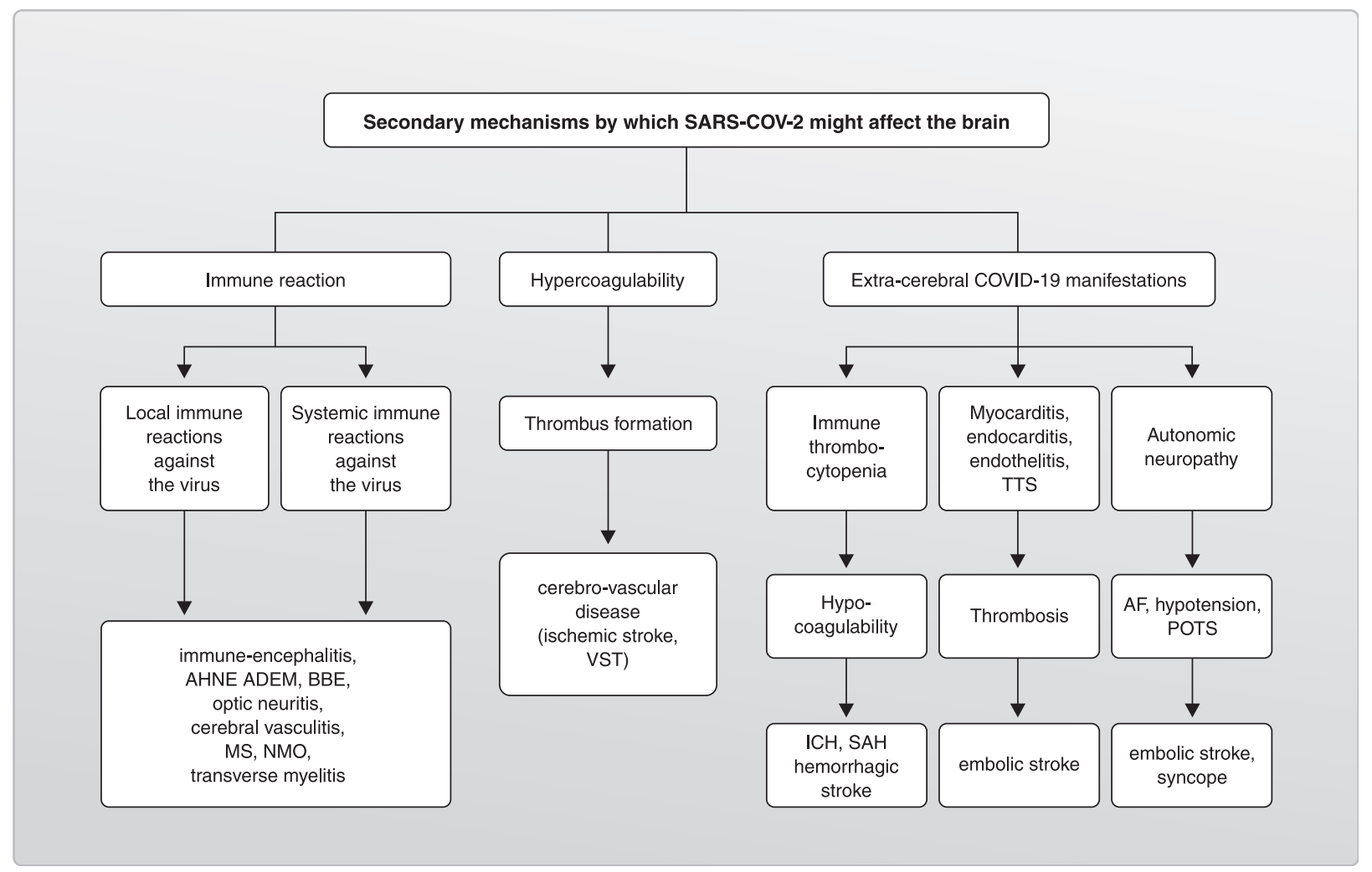

Figure 1 Secondary pathophysiological mechanisms causing cerebral disease in COVID-19. ADEM = acute disseminated encephalomyelitis AF = atrial fibrillation; AHNE = acute hemorrhagic necrotizing encephalitis; BBE = Bickerstaff brainstem encephalitis; COVID-19 = coronavirus disease 2019; ICH = intracerebral hemorrhage; $\mathrm{MS}=$ multiple sclerosis; $\mathrm{NMO}=$ neuromyelitis optica; POTS = postural tachycardia syndrome; SAH = subarachnoid hemorrhage; SARS-CoV-2 = severe acute respiratory syndrome coronavirus 2 ; TTS = Takotsubo syndrome; VST = venous sinus thrombosis.

followed by reduced blood flow, vascular stasis, and vessel occlusion. A third mechanism leading to hypercoagulability could be the activation of several hypoxemia-inducible factors, such as hypoxia-inducible factor $2 \alpha$, which can up- or downregulate several genes involved in physiological hemostasis. A fourth mechanism would be immune thrombocytopenia resulting in dysfunctional thrombocytes. A fifth mechanism explaining the propensity of patients with severe COVID-19 for thrombosis is immobility due to artificial ventilation. Finally, a sixth mechanism would involve the activation of the blood coagulation cascade by the immune response to the virus. Whatever its underlying mechanism, hypercoagulability places COVID-19 patients are at risk of developing VST, which may manifest clinically as seizures, confusion, headache and cervicalgia, neck stiffness, photosensitivity, vertigo, extra-cranial thrombosis (deep venous thrombosis, pulmonary embolism, splanchnic venous thrombosis, portal vein thrombosis, ovarian vein thrombosis, hepatic artery thrombosis, abdominal aortic thrombosis, aortic arch thrombosis), and disseminated intravascular coagulopathy. Even with prophylactic anticoagulant therapy, the incidence of venous thrombosis remains high.

A third pathophysiological mechanism by which SARSCoV-2 can affect the CNS is the heart-brain connection.
It is well appreciated that all organs can be affected by viral infection, including the heart. Cardiac manifestations of SARS-CoV-2 infection include peri- and myocarditis, endocarditis, myocardial infarction, heart failure, arrhythmias, aortic dissection, and Takotsubo syndrome (TTS). Since endocarditis, myocarditis, myocardial infarction, arrhythmias, and TTS can all be complicated by intraventricular thrombus formation, there is an increasing risk of developing embolism of cardiac origin, which, in turn, may lead to ischemic stroke and thus indirect involvement of the CNS by the virus.

A fourth pathophysiological mechanism to explain SARS-CoV-2-associated CNS pathology is the CNS toxicity of certain drugs used in COVID-19. Drugs frequently given to COVID-19 patients which carry neurotoxic potential include steroids, tocilizumab (causes disorientation, aphasia, seizures, cerebral edema), and chloroquine (depresses neuronal activity).

Overall, SARS-CoV-2 may affect the brain directly or indirectly. It is particularly important that secondary mechanisms are not neglected as causes of SARSCoV-2-associated CNS disease. To detect these indirect causes, COVID-19 patients need to be thoroughly and prospectively investigated for multisystem involvement, particularly of the heart, arteries, veins, immune system, and coagulation system. 


\section{Disclosure}

The authors report no conflicts of interest.

\section{References}

1 Finsterer J, Scorza FA. Clinical and pathophysiologic spectrum of neuro-COVID. Mol Neurobiol. 2021;58:3787-91.

2 Ross Russell AL, Hardwick M, Jeyanantham A, White LM, Deb S, Burnside $G$, et al. Spectrum, risk factors and outcomes of neurological and psychiatric complications of COVID-19: a UK-wide cross-sectional surveillance study. Brain Commun. 2021;3:fcab168.

3 Bauer SC, Moral F, Preloger E, Spindler A, Roman M, Logan A, et al. Pediatric COVID-19 delirium: case report of 2 adolescents. WMJ. 2021;120:131-6.

4 Fusunyan M, Praschan N, Fricchione G, Beach S. Akinetic mutism and coronavirus disease 2019: a narrative review. J Acad Consult Liaison Psychiatry. 2021;S2667-960(21)00149-X.

5 Torrico T, Kiong T, D'Assumpcao C, Aisueni U, Jaber F, Sabetian K, et al. Postinfectious COVID-19 catatonia: a report of two cases. Front Psychiatry. 2021;12:696347. 